\title{
NON-COMPLEMENTED OPEN SETS IN EFFECTIVE TOPOLOGY
}

\author{
PHILIP HINGSTON \\ (Received 29 March 1985; revised 30 January 1987) \\ Communicated by J. N. Crossley
}

\begin{abstract}
Notions of effective complementation in effective topological spaces are considered, and several types of non-complemented sets are constructed. While there are parallels with recursively enumerable sets, some unexpected differences appear. Finally, a pair of splitting theorems is proved.
\end{abstract}

1980 Mathematics subject classification (Amer. Math. Soc.): 03 D 45, 54 A 05.

\section{Introduction}

With their paper Recursive Constructions in Topological Spaces [6], Kalantari and Retzlaff introduced the use of recursion theoretic tools in the study of effectiveness in topology. This was the first of a series of papers, [2], [3], [4], [5], and [6]. In these studies recursive properties of open sets form the central theme.

In this paper we develop some ideas suggested by the above work and investigated in the author's Ph.D. thesis [1].

In Section 2 below we give the basic definitions and discuss some notions of effective topological complementation which have been explored.

In Section 3 we go on to consider non-complemented sets, introducing notions of topological creativity and simplicity, and constructing a set which is both simple and creative. Finally we prove a pair of splitting theorems similar to Friedberg's splitting theorem for recursively enumerable sets.

(c) 1988 Australian Mathematical Society $0263-6115 / 88 \$ A 2.00+0.00$ 


\section{Definitions}

We consider a topological space, $X$, with countable basis $\Delta=\left\{\delta_{n}: n \in \omega\right\}$. The elements of $\Delta$ are called basic open sets. We require that every non-empty basic open set contain two disjoint non-empty basic open sets. This condition gives us enough basic open sets to carry out infinite constructions.

We call such a space an effective topological space if there are algorithms by which

$$
\text { given } n, m \in \omega \text { we can find a number } k \text { such that } \delta_{n} \cap \delta_{m}=
$$
$\delta_{k}$,

and

$$
\begin{aligned}
& \text { given } m, n_{1}, \ldots, n_{k} \in \omega \text { we can decide whether or not } \delta_{m} \subseteq \\
& \delta_{n_{1}} \cup \cdots \cup \delta_{n_{k}} .
\end{aligned}
$$

Such a pair of algorithms is called an inclusion algorithm for $X$. This definition is the same as that in [6], except that we do not require conditions I and III given there. Examples of effective topological spaces are given in [1] and [6].

The objects of our study are the r.e. (recursively enumerable) open sets in such an effective topological space. Still following [6], we write

$$
\mathscr{U}_{e}=\bigcup\left\{\delta_{n}: n \in \omega_{e}\right\} \text {, the } e \text { th r.e. open set, }
$$

and

$$
\mathscr{U}_{e}^{s}=\bigcup\left\{\delta_{n}: n \in \omega_{e}^{s}\right\}, \quad \text { where } \omega_{e}^{s} \text { is the finite part of } \omega_{e}
$$

enumerated up to stage $s$ in a standard enumeration of the r.e. sets.

R.e. open sets are the topological analogue of recursively enumerable sets. A natural question presents itself - what is the toplogical analogue of recursive sets? This question is considered in [1], [5] and [6] where several distinct, reasonable answers are found.

If one regards a recursive set as a complemented element of $\mathscr{L}(\omega)$, the lattice of r.e. sets, one arrives at the notion of a complemented r.e. open set, (see [6]), that is an r.e. open set $\mathscr{A}$ for which there is an r.e. open set $\mathscr{B}$ with $\mathscr{A} \cap \mathscr{B}=\varnothing$ and $\mathscr{A} \cup \mathscr{B}$ dense in $X$. One could strengthen this and say that the natural topological complement of $\mathscr{A}$, the exterior of $\mathscr{A}$, written ext $\mathscr{A}$, should be an r.e. open set. Let us call such a set $\mathscr{A}$ strongly complemented (the term $\cap$-complemented is used in [1]).

Or again, one might regard a recursive set as a decidable set. In the topological version one may want to decide which basic open sets are contained in the given r.e. open set, or which basic open sets intersect it nonemptily. In [1], a set for which one can decide the second question is said to be n-recursive. A set for 
which one can decide both questions is called completely resursive in [6]. Clearly $\mathscr{A}$ is completely recursive $\Rightarrow \mathscr{A}$ is $\cap$-recursive $\Rightarrow \mathscr{A}$ is strongly complemented $\Rightarrow \mathscr{A}$ is complemented. Examples constructed in [1], [5] and [6] show that no other implications hold in general, showing that the structure of the lattice of r.e. open sets is substantially different from that of $\mathscr{L}(\omega)$.

In the rest of this paper we investigate non-recursiveness in the topological case.

\section{Creative and simple}

A very useful concept in topological constructions is that of a partition. An r.e. collection $\Gamma=\left\{\gamma_{i}: i \in \omega\right\}$ of basic open sets if called a partition for a set $\mathscr{A}$ if

(i) $\gamma_{i} \neq \varnothing$, for all $i$,

(ii) $i \neq j \Rightarrow \gamma_{i} \cap \gamma_{j}=\varnothing$,

(iii) $\bigcup\left\{\gamma_{i}: i \in \omega\right\} \subseteq \mathscr{A}$, and (iv) $\bigcup\left\{\gamma_{i}: i \in \omega\right\}$ is dense in $\mathscr{A}$.

In the proof of Theorem 3.1 of [5] it is shown that every non-empty r.e. open set possesses a partition. We use this in our Theorem 1 .

In $\mathscr{L}(\omega)$ the set $K=\left\{e: e \in \omega_{e}\right\}$ is a standard example of a non-complemented r.e. set. It is also an example of a creative r.e. set.

Let us say that an r.e. open set, $\mathscr{C}$, is creative if there is an algorithm by which, given an r.e. open set $\mathscr{U}_{e}$ such that $\mathscr{C} \cap \mathscr{U}_{e}=\varnothing$, we can find a non-empty basic open set $\delta_{n}$ such that $\delta_{n} \subseteq \operatorname{ext}\left(\mathscr{C} \cup \mathscr{U}_{e}\right)$. That is, $\delta_{n}$ is an effective witness to $\mathscr{C}$ being non-complemented.

Shi has independently considered creative r.e. open sets and obtained a series of results, but at this time we have seen only an abstract [7]. There he has our Theorem 1, as well as a counterexample to the conjecture that creative r.e. open sets are unique up to isomorphism. We do not know what his definitions and assumptions are.

We first establish the existence of creative sets:

THEOREM 1. Let $X$ be an effective topological space and let $\mathscr{A}$ be a non-empty r.e. open set in $X$. Then $\mathscr{A}$ contains a creative set, $\mathscr{C}$.

Proof. Let $\left\{\alpha_{n}: n \in \omega\right\}$ be a partition for $\mathscr{A}$. Put $\mathscr{C}=\bigcup\left\{\alpha_{e}: \alpha_{e} \cap \mathscr{U}_{e} \neq \varnothing\right\}$. Then if $\mathscr{C} \cap \mathscr{U}_{e}=\varnothing$, we will have $\alpha_{e} \subseteq \operatorname{ext}\left(\mathscr{C} \cup \mathscr{U}_{e}\right)$.

In $\mathscr{L}(\omega)$, creative sets are unique up to isomorphism. We have not been able to prove an analogous theorem for creative sets in an effective topological space, but we have an intermediate result. 
For r.e. open sets $\mathscr{A}, \mathscr{B}$ we say $\mathscr{B}$ is m-reducible to $\mathscr{A}$, and write $\mathscr{B} \leqslant_{m} \mathscr{A}$ if there is a recursive function $f$ such that

$$
\delta_{n} \cap \mathscr{B}=\varnothing \Leftrightarrow \delta_{f(n)} \cap \mathscr{A}=\varnothing .
$$

An open set $\mathscr{A}$ is m-complete if $\mathscr{B} \leqslant \mathscr{A}$ for all r.e. open sets $\mathscr{B}$.

THEOREM 2. Let $X$ be an effective topological space and let $\mathscr{C}$ be a creative r.e. open set in $X$. Then $\mathscr{C}$ is m-complete.

Proof. The algorithm for the creativeness of $\mathscr{C}$ yields a recursive function $h$ such that $\mathscr{C} \cap \mathscr{U}_{e}=\varnothing \Rightarrow \delta_{h(e)} \subseteq \operatorname{ext}\left(\mathscr{C} \cup \mathscr{U}_{e}\right)$.

Let $\mathscr{A}$ be any r.e. open set. Define a recursive function $g$ such that

$$
\mathscr{U}_{g(x, y)}= \begin{cases}\delta_{h(x)} & \text { if } \boldsymbol{\delta}_{y} \cap \mathscr{A} \neq \varnothing, \\ \varnothing & \text { if } \boldsymbol{\delta}_{y} \cap \mathscr{A}=\varnothing .\end{cases}
$$

(Use the intersection algorithm.)

By the recursion theorem, there is a recursive function $n$ such that

$$
\mathscr{U}_{n(y)}=\mathscr{U}_{g(n(y), y)}= \begin{cases}\delta_{h n(y)} & \text { if } \delta_{y} \cap \mathscr{A} \neq \varnothing, \\ \varnothing & \text { if } \delta_{y} \cap \mathscr{A}=\varnothing .\end{cases}
$$

Thus

$$
\begin{aligned}
\delta_{y} \cap \mathscr{A} & \neq \varnothing \Rightarrow \delta_{h n(y)}=\mathscr{U}_{n(y)} \\
& \Rightarrow \delta_{h n(y)} \subseteq \operatorname{ext}\left(\mathscr{C} \cup \mathscr{U}_{n(y)}\right) \\
& \Rightarrow \delta_{h n(y)} \cap \mathscr{C}=\mathscr{U}_{n(y)} \cap \mathscr{C} \neq \varnothing
\end{aligned}
$$

and

$$
\begin{aligned}
\delta_{y} \cap \mathscr{A} & =\varnothing \Rightarrow \mathscr{U}_{n(y)}=\varnothing \\
& \Rightarrow \delta_{h n(y)} \subseteq \operatorname{ext} \mathscr{C} \\
& \Rightarrow \delta_{h n(y)} \cap \mathscr{C}=\varnothing .
\end{aligned}
$$

A creative set is non-complemented, but what other kinds of non-complemented r.e. open sets exist? In $\mathscr{L}(\omega)$ the corresponding question gives rise to simple sets.

One may think of a simple set as one which has a large complement but no infinite r.e. complement, since the only r.e. sets disjoint from it are small. To define an analogous concept in topological terms one needs a notion of "large" and "small". Such a notion is presented in [3], giving rise to the concept of fragmentation simplicity. Here we take a different tack, and try to construct an r.e. open set which meets as many r.e. open sets as possible, while still keeping the 
classical complement "large". If the set to be constructed is to have a non-trivial complement, then certainly there must be at least one basic open set disjoint from it. Unavoidably then, any r.e. open set in which this basic open set is dense is also contained in the complement. If the complement is to be "large" then one would not want this one basic open set to be dense in it, so one is forced to admit another basic open set into the complement, together with any r.e. open set in which any r.e. open set in which these two basic open sets are dense. Surely just two basic open sets should not be dense in the complement, so we must allow another, and so on. This line of thought leads to the following definitions.

A set $\mathscr{A}$ in $X$ is $\Delta$-large if no finite union of basic open sets is dense in $\mathscr{A}$. Otherwise, $\mathscr{A}$ is $\Delta$-small.

Note that this concept is highly dependent on the choice of basis for the topology. To be more precise, let us say that two bases are effectively equivalent if there are algorithms by which, given an enumeration of an r.e. open set in either basis, we can find an enumeration in the other basis for the same open set. It is not difficult to construct examples of spaces, with effectively equivalent bases, in which a set is "large" with respect to one basis but "small" with respect to another. I am grateful to the referee for pointing this out and have taken up his suggestion by using the terms $\Delta$-large, $\Delta$-small and $\Delta$-simple to emphasize this basis dependence.

Observe that any set dense in a $\Delta$-large set is $\Delta$-large and any subset of a $\Delta$-small set is $\Delta$-small.

An r.e. open set $\mathscr{S}$ is called $\Delta$-simple if

(i) ext $\mathscr{S}$ is $\Delta$-large, and,

(ii) $\mathscr{U}_{e} \subseteq \operatorname{ext} \mathscr{S} \Rightarrow \mathscr{U}_{e}$ is $\Delta$-small.

Observe that a $\Delta$-simple set cannot be complemented, since a complement would be $\Delta$-large (being dense in ext $\mathscr{S}$ ) and $\Delta$-small (being a subset of ext $\mathscr{S}$ ).

Note that $X$ may not contain any $\Delta$-large sets. For example, $X$ itself may be an element of $\Delta$. Thus, to construct a $\Delta$-simple set we need $X$ to satisfy some extra condition. It is sufficient to require that $X$ itself is $\Delta$-large, i.e. that no finite union of basic open sets is dense in $X$.

THeOREM 3. Let $X$ be $\Delta$-large. Then $X$ contains a $\Delta$-simple set $\mathscr{S}$.

Proof. We use a finite injury priority construction.

Let $\left\{f_{i}: i \in \omega\right\}$ be an effective list of all finite unions of basic open sets. We aim to ensure that, for all $i \in \omega, \mathscr{S} \cup f_{i}$ is not dense in $X$. From this it follows that ext $\mathscr{S}$ is $\Delta$-large. To do this we choose a witness $B_{i}$ for each $i$, a basic open set, which we try to keep disjoint from $\mathscr{S} \cup f_{i}$. 
We also try to make every $\mathscr{U}_{e}$ intersect $\mathscr{S}$ non-trivially. It turns out that we can fail only if $\mathscr{U}_{e}$ is $\Delta$-small, so any r.e. open subset of $X-\mathscr{S}$ will be $\Delta$-small.

Construction. Our requirement are

$$
\begin{aligned}
& N_{e}: B_{e}=\lim B_{e}^{s} \text { exists and is non-empty, } \\
& P_{e}: \mathscr{U}_{e} \text { is } \Delta \text {-large } \Rightarrow \mathscr{U}_{e} \cap \mathscr{S} \neq \varnothing .
\end{aligned}
$$

We give these the priority ranking $N_{0}>P_{0}>N_{1}>P_{1}>\cdots$. We say that $N_{e}$ requires attention at stage $s$ if $B_{e}^{s}$ is not defined. $N_{e}$ is attacked at stage $s$ by finding the first $n$ such that $\delta_{n} \neq \varnothing$ and $\delta_{n} \cap\left(\mathscr{S}^{s} \cup f_{e}\right)=\varnothing$, and putting $B_{e}^{s+1}=\delta_{n}$. Note that such a $\delta_{n}$ exists since $X$ is $\Delta$-large.

$N_{e}$ is injured at stage $s$ if $B_{e}^{s}$ is defined and $B_{e}^{s+1}$ is undefined. We say that $P_{e}$ requires attention at stage $s$ if $\mathscr{U}_{e}^{s} \cap \mathscr{S}^{s}=\varnothing$, and for some $n \leqslant s, \delta_{n} \subset \mathscr{U}_{e}^{s}$ and $\delta_{n} \cap \bigcup\left\{B_{i}^{s}: i \leqslant e\right.$ and $B_{i}^{s}$ is defined $\}=\varnothing . P_{e}$ is attacked at stage $s$ by finding the least such $n$ and putting $\mathscr{S}^{s+1}=\mathscr{S}^{s} \cup \delta_{n}, B_{i}^{s+1}$ undefined for all $i>e$.

Stage 0 . Set $\mathscr{S}^{0}=\varnothing, B_{e}^{0}$ is undefined for all $e \in \omega$.

Stage $s>0$. Find the highest priority requirement requiring attention at this stage and attack it.

\section{End of construction.}

The usual argument shows that all the $N_{e}$ are met. Clearly, if $P_{e}$ is ever the least requiring attention, then $P_{e}$ is met. On the other hand, if $\mathscr{U}_{e} \cap \mathscr{S}=\varnothing$ and $P_{e}$ is never the least requiring attention, then $B_{0} \cup \cdots \cup B_{e}$ is dense in $\mathscr{U}_{e}$ so that $\mathscr{U}_{e}$ is $\Delta$-small. Thus the $P_{e}$ are all met and $\mathscr{S}$ is $\Delta$-simple as claimed.

In $\mathscr{L}(\omega)$, the complement of a creative set contains an infinite r.e. set, so a creative set is not simple, but the analogous argument does not go through for creative sets. Let $\mathscr{C}$ be a creative set. Specifically, since $\varnothing \subseteq X-\mathscr{C}$ we can find a basic open set, $\alpha_{1}$, such that $\alpha_{1} \subseteq X-(\mathscr{C} \cup \varnothing)$. We can then find $\alpha_{2} \subseteq X-$ $\left(\mathscr{C} \cup \alpha_{1}\right), \quad \alpha_{3} \subseteq X-\left(\mathscr{C} \cup \alpha_{1} \cup \alpha_{2}\right)$, etc. But the infinite r.e. collection $\left\{\alpha_{1}, \alpha_{2}, \alpha_{3}, \ldots\right\}$ may not have an $\Delta$-large union. This is shown clearly in the next theorem.

TheOREM 4. Let $X$ be $\Delta$-large. Then $X$ contains a $\Delta$-simple, creative open set $\mathscr{P}$.

Proof. We modify the construction in Theorem 3.

Let $A$ be a fixed basic open set and let $A_{0}, A_{1}, \ldots$ be a partition for it. To ensure that $\mathscr{P}$ is creative, we require that, for all $e, \mathscr{P} \cap \mathscr{U}_{e}=\varnothing \Rightarrow A_{e} \subseteq X-$ $\left(\mathscr{P} \cup \mathscr{U}_{e}\right)$. Let us call this requirement $Q_{e}$. 
We say that $Q_{e}$ requires attention at stage $s$ if $\mathscr{U}_{e}^{s} \cap \mathscr{P}^{s}=\varnothing$ and $\mathscr{U}_{e}^{s} \cap A_{e} \neq \varnothing$. $Q_{e}$ is attacked at stage $s$ by putting $\mathscr{P}^{s+1}=\mathscr{P}^{s} \cup A_{e}$.

The following changes need to be made.

$N_{e}$ is attacked at stage $s$ by finding the first $n$ such that $\delta_{n} \cap\left(\mathscr{P}^{s} \cup A \cup f_{e}\right)=$ $\varnothing$, and putting $B_{e}^{s+1}=\delta_{n}$.

$P_{e}$ requires attention at stage $s$ if $\mathscr{U}_{e}^{s} \cap \mathscr{P}^{s}=\varnothing$, and for some $n \leqslant s, \delta_{n} \subseteq \mathscr{U}_{e}^{s}$ and $\delta_{n} \cap\left(A \cup \cup\left\{B_{i}^{s}: i \leqslant e\right.\right.$ and $B_{i}^{s}$ defined $\left.\}\right)=\varnothing$. We give these the priority ranking $N_{0}>P_{0}>Q_{0}>N_{1}>P_{1}>Q_{1} \cdots$.

The same arguments as before show that the $N_{e}$ and $P_{e}$ are all met. Suppose $\mathscr{P} \cap \mathscr{U}_{e}=\varnothing$. If $Q_{e}$ ever requires attention it will eventually be the least requiring attention and so will be attacked. Then $A_{e} \subseteq \mathscr{P}$ and $A_{e} \cap \mathscr{U}_{e} \neq \varnothing$ imply $\mathscr{P} \cap \mathscr{U}_{e} \neq \varnothing$. This is a contradiction so $Q_{e}$ never requires attention, that is, $\mathscr{U}_{e} \cap A_{e}=\varnothing$ as claimed.

An interesting question which we have not been able to answer, even for particular $X$, is whether there exist sets which are " $\Delta$-maximal", where this is defined in the same spirit as $\Delta$-simple. That is, let us call an r.e. open set $\mathscr{M} \Delta$-maximal if

$$
\text { ext } \mathscr{M} \text { is } \Delta \text {-large }
$$

and

$$
\left.\begin{array}{rl}
\mathscr{U}_{e} \supseteq \mathscr{M} \Rightarrow \mathscr{M} \cup f \text { is dense in } \mathscr{U}_{e} \\
\text { or } \mathscr{U}_{e} \cup f \text { is dense in } X
\end{array}\right\} \quad \begin{aligned}
& \text { for some finite union, } \\
& f, \text { of basic open sets. }
\end{aligned}
$$

We conclude with a topological version of Friedberg's splitting theorem.

THEOREM 5. Let $X$ be an effective topological space. Let $\mathscr{A}$ be an r.e. open set in $X$ which is not complemented. Then $\mathscr{A}$ contains r.e. open subsets $\mathscr{B}_{1}$ and $\mathscr{B}_{2}$ such that $\mathscr{B}_{1} \cap \mathscr{B}_{2}$ is dense in $\mathscr{A}, \mathscr{B}_{1} \cap \mathscr{B}_{2}=\varnothing$, and neither $\mathscr{B}_{1}$ not $\mathscr{B}_{2}$ is complemented.

Proof. Let $\left\{\alpha_{n}\right\}_{n \in \omega}$ be a full enumeration of $\mathscr{A}$. That is, for some $e \in \omega$, $\mathscr{A}=\mathscr{U}_{e}$ and $\left(\delta_{i} \subseteq \mathscr{U}_{e}^{s}\right.$ for some $s \in \omega \Leftrightarrow \delta_{i}=\alpha_{n}$ for some $\left.n \in \omega_{e}\right)$.

Construction. We construct $\mathscr{B}_{1}$ and $\mathscr{B}_{2}$ in stages. Set $\mathscr{B}_{1}^{0}=\mathscr{B}_{2}^{0}=\varnothing$.

Stage $s+1$. Let $\alpha=$ the first $\alpha_{n}$ such that $\alpha_{n} \neq \varnothing$ and $\alpha_{n} \cap\left(\mathscr{B}_{1}^{s} \cup \mathscr{B}_{2}^{s}\right)=\varnothing$. Let $x_{s}=$ the least $x \leqslant s$ such that $\alpha \cap \mathscr{U}_{x}^{s} \neq \varnothing$ and either $\mathscr{U}_{x}^{s} \cap \mathscr{B}_{1}^{s}=\varnothing$ or $\mathscr{U}_{x}^{s} \cap \mathscr{B}_{2}^{s}=\varnothing$. Effectively find some $\beta \subseteq \alpha \cap \mathscr{U}_{x_{s}}^{s}$.

$\operatorname{Case}(\mathrm{i}) . \mathscr{U}_{x_{s}}^{s} \cap \mathscr{B}_{1}^{s}=\varnothing$. Put $\mathscr{B}_{1}^{s+1}=\mathscr{B}_{1}^{s} \cup \beta, \mathscr{B}_{2}^{s+1}=\mathscr{B}_{2}^{s}$. 
Case (ii). $\mathscr{U}_{x_{s}}^{s} \cap \mathscr{B}_{1}^{s} \neq \varnothing$ and $\mathscr{U}_{x_{s}}^{s} \cap \mathscr{B}_{2}^{s}=\varnothing$. Put $\mathscr{B}_{1}^{s+1}=\mathscr{B}_{1}^{s}, \mathscr{B}_{2}^{s+1}=\mathscr{B}_{2}^{s} \cup$ $\beta$. If no such $x$ exists, put $\mathscr{B}_{1}^{s+1}=\mathscr{B}_{1}^{s} \cup \alpha, \mathscr{B}_{2}^{s+1}=\mathscr{B}_{2}^{s}$.

At the end of time put $\mathscr{B}_{1}=\cup \mathscr{B}_{1}^{s}$ and $\mathscr{B}_{2}=\cup \mathscr{B}_{2}^{s}$.

\section{End of construction}

By construction, $\mathscr{B}_{1} \cap \mathscr{B}_{2}=\varnothing$. To see that $\mathscr{B}_{1} \cup \mathscr{B}_{2}$ is dense in $\mathscr{A}$, suppose not and that $\delta \subseteq \mathscr{A}$ is disjoint from $\mathscr{B}_{1} \cup \mathscr{B}_{2}$. There exists $n$ such that $\alpha_{n} \subseteq \delta$. By assumption, $\alpha_{n}$ is disjoint from $\mathscr{B}_{1}^{s} \cup \mathscr{B}_{2}^{s}$ for all $s$. Thus at some stage $s, \alpha_{n}$ is the first such. At this stage, by construction, $\alpha_{n} \cap\left(\mathscr{B}_{1}^{s+1} \cup \mathscr{B}_{2}^{s+1}\right) \neq \varnothing$. This is a contradiction, so $\mathscr{B}_{1} \cup \mathscr{B}_{2}$ is dense in $\mathscr{A}$, as claimed.

It remains to show that $\mathscr{B}_{1}$ and $\mathscr{B}_{2}$ are not complemented. Suppose, for a contradiction, that $\mathscr{B}_{1}$ is complemented. We show that in this case $\mathscr{A}$ is complemented.

Suppose $\mathscr{B}_{1}$ has a complement, $\mathscr{U}_{m}$. Let $s_{0}$ be the least $s>m$ such that if $k<m, t>s$, then $x_{t} \neq k$ (such a stage exists since each $k=$ some $x_{t}$ at most twice during the construction).

Suppose that at some stage $s>s_{0}$, and for some basic open set $\delta$ we have $\delta \subseteq \mathscr{U}_{m}^{s}$ and $\delta \cap \mathscr{B}_{2}^{s}=\varnothing$. We claim that in this case $\delta \cap \mathscr{B}_{2}=\varnothing$. Suppose otherwise and that $t+1$ is the earliest stage such that $\delta \cap \mathscr{B}_{2}^{t+1} \neq \varnothing$. So $t \geqslant s$. Then $\mathscr{B}_{2}^{t+1}=\mathscr{B}_{n}^{t} \cup \beta$ where $\beta \cap \delta \neq \varnothing$. So $t \geqslant s$. Then $\mathscr{B}_{2}^{t+1}=\mathscr{B}_{2}^{t} \cup \beta$ where $\beta \cap \delta \neq \varnothing$. Thus $\beta \cap \mathscr{U}_{m}^{t} \neq \varnothing$. But since $t>s_{0}$, we must have $x_{t}=m$. However, this would be a Case (i) situation, and then $\mathscr{B}_{1}^{t+1}=\mathscr{B}_{1}^{t} \cup \beta$, contradicting the fact that $\delta \cap \beta_{1}=\varnothing$. Therefore, the claim holds. Thus

$\mathscr{C}=\bigcup\left\{\delta_{n}: \delta_{n} \subseteq \mathscr{U}_{m}^{s}\right.$ and $\delta_{n} \cap \mathscr{B}_{2}^{s}=\varnothing$ for some $\left.s \geqslant s_{0}\right\} \subseteq$ ext $\mathscr{A}$. We claim that $\mathscr{C}$ is a complement for $\mathscr{A}$. To see this, suppose $\delta \subseteq$ ext $\mathscr{A}$. Thus $\delta \subseteq$ ext $\mathscr{B}_{1}$, and so $\delta \cap \mathscr{U}_{m} \neq \varnothing$. Then there is some $\gamma \subseteq \delta \cap \mathscr{U}_{m}^{\prime}$ at some stage $t$ and so $\gamma \subseteq \mathscr{C}$ and we get $\gamma \cap \delta \neq \varnothing$. Thus $\mathscr{C} \cup \mathscr{A}$ is dense in $X$, and $\mathscr{C}$ is a complement as claimed.

This contradiction shows that $\mathscr{B}_{1}$ is not complemented and a similar argument shows that $\mathscr{B}_{2}$ is not complemented.

CoRollary. Let X be an effective topological space. Let $\mathscr{A}$ be an r.e. open set in $X$ which is not $\cap$-recursive. Then $\mathscr{A}$ contains r.e. open sets $\mathscr{B}_{1}$ and $\mathscr{B}_{2}$ such that $B_{1} \cup \mathscr{B}_{2}$ is dense in $\mathscr{A}, \mathscr{B}_{1} \cap \mathscr{B}_{2}=\varnothing$ and neither $\mathscr{B}_{1}$ nor $\mathscr{B}_{2}$ is $\cap$-recursive.

Proof. The construction is the same as in the theorem. As before, $\mathscr{C}=\bigcup\left\{\delta_{n}\right.$ : $\delta_{n} \subseteq \mathscr{U}_{m}^{s}$ and $\delta_{n} \cap \mathscr{B}_{2}^{s}=\varnothing$ for some $\left.s \geqslant s_{0}\right\} \subseteq$ ext $\mathscr{A}$. Here we can assume that ext $\mathscr{B}_{1}=\mathscr{U}_{m}$ where $\omega_{m}=\left\{n: \delta_{n} \subseteq\right.$ ext $\left.\mathscr{B}_{1}\right\}$ is recursive. Thus if $\delta \subseteq$ ext $\mathscr{A}$, then at some stage $s \geqslant s_{0}$ we will have $\delta \subseteq \mathscr{U}_{m}^{s}$ and $\delta \cap \mathscr{B}_{2}^{s}=\varnothing$. 
Therefore $\left\{n: \delta_{n} \subseteq \mathscr{U}_{m}^{s}\right.$ and $\delta_{n} \cap \mathscr{B}_{2}^{s}=\varnothing$ for some $\left.s \geqslant s_{0}\right\}$ is equal to $\{n$ : $\delta_{n} \cap \mathscr{A}=\varnothing$ \}. The first set is clearly r.e., the second is co-r.e. and so $\mathscr{A}$ is ก-recursive.

As before, this contradiction shows that $\mathscr{B}_{1}$ cannot be $\cap$-recursive and similarly $\mathscr{B}_{2}$ is not $\cap$-resursive.

\section{References}

[1] $\mathrm{P}$. F. Hingston, Effectiveness in rings and topology, $(\mathrm{Ph} . \mathrm{D}$. Thesis, Monash University, November 1983).

[2] I. Kalantari, Major subsets in effective topology. Patras Logic Symposion (Patras, 1980), pp. 77-94 (Stud. Logic Foundations Math., 109, North-Holland, 1982).

[3] I. Kalantari and A. Leggett. 'Simplicity in effective topology.' J. Symbolic Logic 47 (1982), 169-183.

[4] I. Kalantari and A. Leggett. 'Maximality in effective topology.' J. Symbolic Logic 48 (1983), $100-112$.

[5] I. Kalantari and J. B. Remmel. Degrees of recursively enumerable topological spaces. $J$. Symbolic Logic 48 (1983), 610-622.

[6] I. Kalantari and A. Retzlaff. 'Recursive constructions in topological spaces.' J. Symbolic Logic 44 (1979), 609-625.

[7] N. Shi, Unpublished abstract.

\section{Department of Mathematics}

Monash University

Clayton, Victoria 3168

Australia 